the polypeptide distribution in adults is highly variable between individuals.

A separate analysis of the polypeptide patterns of 13 infants who had undergone surgery for UPJO, or whose UPJO had resolved spontaneously, yielded interesting results. Although 11 of these infants were clinically normal, none had recovered the 'healthy' neonatal polypeptide pattern. Longer-term follow-up was recommended to see if such infants eventually recover normal patterns.

Original article Decramer S et al. (2006) Predicting the clinical outcome of congenital unilateral ureteropelvic junction obstruction in newborn by urinary proteome analysis. Nat Med 12: 398-400

\section{Intraprostatic injection of botulinum toxin type A could relieve BPH symptoms}

The bladder-outlet obstruction associated with benign prostatic hyperplasia $(\mathrm{BPH})$ has both a static and dynamic component; dynamic obstruction is caused by contraction of smooth muscle within the prostate. Botulinum A toxin (BTX) is known to block neurotransmitter release in autonomic neurons (preferentially those with cholinergic receptors), and animal studies have shown that intraprostatic injections of BTX cause prostate denervation and atrophy. Chuang et al. investigated the histologic effects of BTX in a canine model of BPH, and also performed a pilot study of intraprostatic BTX injection in men with symptomatic BPH who had an unacceptable reaction to $\alpha$-blocker therapy (in terms of clinical response or adverse effects).

Intraprostatic injections of $4 \mathrm{ml}$ saline with or without (control group) $100 \mathrm{U}$ of BTX were given to eight dogs ( $2 \mathrm{ml}$ to each lobe). Histologic examination of prostates harvested 1 or 3 months after BTX injection revealed that treated glands showed substantially more atrophy and diffuse apoptosis than controls. The authors are carrying out further histologic examination of the samples to explore which neuropeptides are involved in this process.

In the second part of the study, $200 \mathrm{U}$ of BTX in $8 \mathrm{ml}$ saline were given to eight men with $\mathrm{BPH}$ ( $4 \mathrm{ml}$ to each prostatic lobe). At 1 month followup, the men showed marked improvements in symptom and quality-of-life scores, reduced prostate volume, and increased maximal urine flow rate, compared to baseline values.
These effects remained after 3 months, and no adverse events occurred.

Original article Chuang Y-C et al. (2006) Intraprostatic injection of botulinum toxin type-A relieves bladder outlet obstruction in human and induces prostate apoptosis in dogs. BMC Urology [doi:10.1186/1471-2490-6-12]

\section{Is hypercholesterolemia linked to prostate cancer?}

A new study from Italy suggests that men with prostate cancer are 1.5 times more likely, overall, to have had hypercholesterolemia than men without prostate cancer.

Bravi and colleagues' hospital-based, casecontrol study compared 1,294 men admitted with incident prostate cancer and 1,451 control men (both groups aged 46-74 years), admitted to the same Italian hospitals for acute, noncancer conditions (excluding urologic or hormonal disorders). During an interview in hospital, participants provided information on their sociodemographic characteristics, food, tobacco, and alcohol consumption, and family history of prostate cancer. They also provided recalled histories (including age at first diagnosis or treatment) in relation to 10 medical conditions.

After adjustment for confounding factors, hypercholesterolemia was the only condition included in the survey that was markedly associated with prostate cancer. The link between these factors was strongest in men who were diagnosed with cancer aged 46-50 or 6574 years - these men were nearly twice as likely as similar-aged controls to have had hypercholesterolemia. The authors also report a link between gallstones and prostate cancer, which was most evident in lean men (BMl $\left.<26 \mathrm{~kg} / \mathrm{m}^{2}\right)$. This trend did not reach significance, however, perhaps because low numbers of patients were involved. Nonetheless, Bravi et al. think that this finding could be relevant, because the biological mechanisms underlying hypercholesterolemia and gallstone formation are similar.

Further investigation of the link between hypercholesterolemia and prostate cancer is needed, particularly in studies using a healthy (not hospitalized) control population and objective methods of assessment, rather than self-reported patient histories.

Original article Bravi F et al. (2006) Self-reported history of hypercholesterolaemia and gallstones and the risk of prostate cancer. Ann Oncol 17: 1014-1017 